\title{
Librarianship and the Culture of Busy
}

Pam Ryan

University of Alberta Libraries

Denise Koufogiannakis

University of Alberta Libraries

There are not enough hours in a day. We're busy, we're pulled in many directions, our attention is needed on so many issues by so many people, and we're worried about losing our life-balance. This is a fact of life for all of us. This is our environment. Realists know that this is not going away any time soon and that the only solution is to take personal responsibility for managing the busyness of our lives.

This constant state of "busy" has entered the fabric of our daily work culture in ways that are damaging to our organizations. Busyness is an increasingly acceptable way to (misguidedly) self-promote or seek recognition, and is used as an excuse to deflect important work and impede progress. The results are resentment among peers and damage to professional reputations.

Does this situation sound familiar? You are in a meeting, teleconference, or email discussion and need someone to volunteer to do something. Volunteersilence ensues and the battle begins.

"I just can't take that on right now. I have to do A, B, and C. I'm just so busy."

"Tell me about it, I have $X, Y, Z$ to complete."

"Oh, you can't even understand how busy I am..."

"You think that's bad...."

So, the battle of one-upmanship continues, taking up precious time, detracting from the issues at hand and increasingly stopping progress in its tracks.

Librarians engage in this battle for superiority, based not on individual accomplishments, we're far too modest for that, but rather on one's "volume of busy". The point of this battle is to prove that we do more and have less free time than our peers, and are thus more important. We have so much on our plates, we cannot possibly take on another thing, so we are increasingly forgiven from additional contribution by nature of our busy excellence.

We need to change this. We need to assume that all of our colleagues are busy, productive, and professionally engaged people and stop the dialogue of busy as a badge of honour. Bemoaning your busy state or seeking stress-sympathy with a colleague for a moment of stress-reduction in an offline conversation is exceedingly normal. Constantly doing so in work situations is unprofessional and shows lack of respect for your colleagues' contributions. When you complain 
about being busy, you simply look like someone who wants to complain. When you use how busy you are as a constant excuse or topic of professional conversation, you are not gaining anyone's respect or admiration, you are simply giving permission for others to exhibit similar behaviour by making it acceptable in your workplace culture.

What are other hazards of cultivating an "I'm so busy" work persona? You may be perceived as:

- out-of-control, frenzied, and lacking time management skills;

- not able to quickly shift gears as needed or judge what are important new priorities that need attention;

- inflexible, rigid or focused only on personally-defined areas of importance;

- a work-deflector, not a team player, not someone to rely on; and

- not innovative; a progress-impeder.

This can result in missed opportunities and professional pigeon-holing.

\section{Eliminate the culture of busy. What you can do.}

--When Chairing meetings, if "too-busy" conversation begins, try to regain focus by noting that everyone is busy but that progress needs to be made. If people on your team are not willing to contribute, it is time to find new members who will. Make active contribution an expectation from the start.

--Don't volunteer to serve on committees unless you are able to contribute real work. It's not professional to put something on your resume when your contribution amounts to meeting attendance. Your peers are well aware of your contribution.

-- If you are truly overwhelmed, as we all can be at some point, don't volunteer in the first place. A simple, "I'm sorry, I can't take that on right now" will suffice. No need to waste your colleagues' time with the minute details of what is making you too busy.

--Reflect. Examine what you are doing and determine how you can restructure. We all go through periods of wheel-spinning but we each have the responsibility to pull it back into manageable shape. If something is important to you, make the time for it. If you are spending time on low-impact tasks, enjoy the jettisoning of them.

-- Be honest. Talk about this issue in your workplace. Has complaint taken the place of possibility? Be the change; model the behaviours that make our workplaces hopeful, positive, enthusiastic and productive environments. 
Partnership: the Canadian Journal of Library and Information Practice and Research, vol. 2, no. 1 (2007)

Pam and Denise are both librarians at the University of Alberta. They try to never be too busy to write on topics of interest to them. 